\title{
Therapeutic Success in Amoebic and Pyogenic Liver Abscesses
}

\author{
Azhari Gani ${ }^{1}$, Iskandar Zakaria ${ }^{2}$ \\ 1,2Departement of Internal Medicine, Faculty of Medicine, Universitas Syiah Kuala, Banda Aceh, \\ Indonesia \\ Email: azharigani@unsyiah.ac.id
}

\begin{abstract}
:
A 52 year old male patient presented with complaints of upper right abdominal pain since 1 month ago, worsening in the last 1 day. Fever has been complained of since 1 month ago, fever fluctuates indefinitely, comes down with fever-reducing drugs, complained of weakness. There is nausea and vomiting, vomiting 1-2 times per day, black vomit like coffee is not there. Complained about weight loss. BAK is like strong tea. Liquid defecation has been complained of since 5 weeks ago, initially liquid defecation was accompanied by mucus and blood with a frequency of 3-4 times per day for 3 days, at that time the patient went to the health center and complaints were reduced and now liquid CHAPTER 1-2 times a day is yellow, liquid, no mucus, no blood for the past 1 month. The patient is an agricultural instructor with his daily activities going down to the farm. History. History of diabetes mellitus denied. Patients diagnosed with multiple pyogenic liver abscess were treated at RSUDZA and received bed rest therapy, $1800 \mathrm{kcal} /$ day soft food diet, IVFD NaCl 0.9\% 20 drops per macro minute, metronidazole drip $500 \mathrm{mg}$ every 8 hours, ciprofloxacin $2 x 500 \mathrm{mg}$, intravenously paracetamol 1 gram every 8 hours. The patient was treated for 17 days, on the 10th day of hospitalization the patient was performed a laparotomy and multiple incisions of the liver abscess, purulent abscess fluid mixed with blood. The abscess fluid was cultured as a result of Entamoeba Colli, and metronidazole antibiotic therapy was continued. During treatment, the patient experienced clinical improvement, the patient was opened surgical sutures on the 10th and 15th day after laparotomy, the surgical wound improved, the patient experienced clinical improvement, currently the patient is still a control at the Internal Medicine and Surgery Department at Cut Nyak Dhien Meulaboh Hospital.
\end{abstract}

Keywords:

therapeutic; amoebic; pyogenic liver abscesses

\section{Introduction}

Liver abscess is an infectious lesion of the liver. There are 2 types of liver abscess, the most common are pyogenic abscess and amoebic abscess. Pyogenic liver abscess is a rare but potentially fatal case, with a reported incidence of 20 per 100,000 patients in hospital populations in western countries. The severity depends on the source of the infection and the patient's condition. Amoebic liver abscess is most commonly found in tropical areas, especially "Entamoeba histolytica" which is endemic and its prevalence is greatest in individuals (mostly young men) with decreased or suppressed immune systems (Dutta, A. 2012; Nattakom, S. 2017).

Over the past 50 years, there has been a major shift in the causes of pyogenic liver abscess. Pyogenic liver abscessmany caused by hematogenous spread or through gastrointestinal infectionascending infection of the biliary. Ascending infection of the biliary is secondary to the most common obstruction causing pyogenic liver abscess. The etiology of bilirary obstruction has several differences based on geographic location: in Western countries 
most patients with malignant disease while in Asia the most common causes of pyogenic liver abscess are gallstones and hepatolithiasis, between $15-55 \%$ of patients have no known cause of pyogenic liver abscess (called cryptogenic). Pyogenic liver abscess does not show a sexual predilection but occurs most frequently in middle aged patients (Dutta, A. 2012, Singh, S. 2013; Mortele, K.J. 2004).

Amoebic liver abscess is the most common part of extra-intestinal infection of amubiasis, but the incidence is less than $1 \%$ in Entamoeba histolytica infection. It is estimated that the prevalence of amoebic liver abscess in the world is around 50 million infections per year. Amoebic liver abscess is 3-10 times more common in men. Most patients with amoebic liver abscess are aged 20-40 years with a history of staying or traveling or emigration from endemic areas. The occurrence of an amoebic liver abscess in people who do not travel to endemic areas, or live in endemic areas, raises the suspicion of immunosuppression, especially Acquired Immunodeficiency Syndrome (AIDS). Host factors that contribute to disease severity are youth, pregnancy, malnutrition, alcoholism, use of glucocorticoids, and malignancy. Amoebic liver abscess lesions are usually single and are found mostly in the right lobe of the liver. The incidence of amoebic liver abscess in the left lobe of the liver is $5 \%$ $21 \%$ of cases. Liver abscess has a thin-walled capsule with a central necrotic consistency of thick fluid. Usually, the abscess fluid is odorless, like "chocolate syrup", the mortality rate is estimated to be around $0.2-2.0 \%$ of adults and 26\% in children (Dutta, A. 2012; Singh, S. 2013; Waleleng, B. 2014; Sharma, M.P. 2003; Chiari, B. 2003).

Both amoebic and pyogenic liver abscesses are a major cause of morbidity and mortality in tropical countries. In the Democratic Republic of Congo (DRC), almost the entire population is affected at some point in their lives and the high prevalence of this parasitosis is one of the many factors contributing to its resistance (Guillaume in Christian, 2020). However, several interventional advances in radiology, intensive care, advances in antibiotic therapy, and the use of sonography and computerized tomography scanning of the abdomen have made earlier diagnosis and treatment of patients with liver abscesses, thereby increasing patient cure rates.Early diagnosis and percutaneous imagingguided drainage have reduced the mortality rate (from $40 \%$ of cases to $2 \%$ ). Percutaneous drainage is required in cases where the abscess is relatively small $(5 \mathrm{~cm}$ in diameter) and given a single antibody to give success.5. The initial antibiotic regimen should include metronidazole for 6 weeks until an amoebic liver abscess can be serologically ruled out. Percutaneous drainage of liver abscess is important in advances in the treatment of pyogenic liver abscess 34.

\section{Case Report}

\subsection{Case Report I}

A 52 year old male patient presented with complaints of upper right abdominal pain since 1 month ago, worsening in the last 1 day. Fever has been complained of since 1 month ago, fever fluctuates indefinitely, comes down with fever-reducing drugs, complained of weakness. There is nausea and vomiting, vomiting 1-2 times per day, black vomit like coffee is not there. Complained about weight loss. BAK is like strong tea. Liquid defecation has been complained of since 5 weeks ago, initially liquid defecation was accompanied by mucus and blood with a frequency of 3-4 times per day for 3 days, at that time the patient went to the health center and complaints were reduced and now liquid CHAPTER 1-2 times a day is yellow, liquid, no mucus, no blood for the past 1 month. The patient is an agricultural instructor with his daily activities going down to the farm. History. History of diabetes mellitus denied. 
On vital examination, compos mentis awareness was found, blood pressure 110/70 $\mathrm{mmHg}$, pulse rate 84 times per minute, breath frequency 24 times per minute, visual analogue score3-4, body mass index underweight. On physical examination, the eyes of the conjunctiva of the inferior palpebra were pale and absent, the icteric sclera was absent. In the neck, the jugular vein pressure was normal, lymph node enlargement was absent. In the lungs, both lungs were found to be symmetrical, no retraction, vesicles in both lung fields, rhonchi and wheezing were not found in both lung fields. In the abdomen, there is a soepol, the liver is palpable $5 \mathrm{~cm}$ below the right rib arch, $6 \mathrm{~cm}$ below the xiphoid process, as if there is pressure, flat surface, chewy consistency, blunt edges, spleen and renal are not palpable, tympanic and peristalsis are present. In all four limbs normal.

On laboratory examination found, hemoglobin $10.1 \mathrm{~g} / \mathrm{dl}$, leuk ocytes 15,000 / $\mathrm{mm}^{3}$, platelets 383,000/ mm3, segment neutrophils $80 \%$, urea $50 \mathrm{mg} / \mathrm{dl}$, creatinine $0.6 \mathrm{mg} / \mathrm{dl} /$, albumin $3.46 \mathrm{mg} / \mathrm{dl}$, SGOT $28 \mathrm{U} / \mathrm{L}$, SGPT $21 \mathrm{U} / \mathrm{L}$, direct bilirubin 0, $20 \mathrm{mg} / \mathrm{dl}$, indirect $0.50 \mathrm{mg} / \mathrm{dl}$, KGDS $101 \mathrm{mg} / \mathrm{dl}$, negative HBsAg, sodium $132 \mathrm{mmol} / \mathrm{L}$, potassium 3.8 mmol / L, chloride $91 \mathrm{mmol} / \mathrm{L}$. On abdominal ultrasound examination of the liver, multiple mixed echoic lesions were found in the right and left lobe of the liver, suggesting a liver abscess. On abdominal CT scan, the impression was that of a liver abscess measuring $16.5 \mathrm{~cm}$.

Patients diagnosed with amoebic liver abscess, treated for 20 days of treatment received bed rest therapy, liver diet $1700 \mathrm{kcal} /$ day, IVFD NaCl 0.9\% 20 drops per minute, intravenouslyceftriaxone $2 \mathrm{~g} / 24$ hours, metronidazole drip $500 \mathrm{mg} / 8$ hours, intravenous ondancetron $4 \mathrm{mg} / 8$ hours, coditam tablets $3 \times 10 \mathrm{mg}$. The patient was drained of the liver abscess by digestive surgery on the 7 th day of admission, $200 \mathrm{cc}$ of dark red discharge from the drainage on the first day, $50 \mathrm{cc}$ on the second day. The results of the liver abscess drainage fluid culture showed Entamoeba Hystolitica, antibiotic therapy was continued until the 20th day, the patient during treatment experienced clinical improvement and found no complications. The patient then went to an outpatient treatment, control to the disease department in the gastroenterohepatology and digestive surgery division, the patient experienced significant improvement.

\subsection{Case Report II}

The 41 year old male patient presented with complaints of upper right abdominal pain since 3 months of weight 1 week before admission to the hospital. Complained of fever for 3 months is intermittent. Nausea was found, vomiting was found 1-2 times per day. Decreased appetite was found, weight loss was found, $4 \mathrm{~kg}$ in the last 3 months. BAK smooth there are no complaints, no black CHAPTER, no liquid CHAPTER. The patient is a trader who is also a farmer. The patient 2 months ago was treated with the same complaint and was diagnosed with a liver abscess. Patient with liver abscess measuring $6 \mathrm{~cm}$. The patient initially did not want to be operated on so he was sent home with antibiotics and then the control patient went to the Internal Medicine Department of RSUDZA. After 1.5 months of outpatient control patients, fever returned, the upper right abdominal pain was getting worse so that the patient was again treated. History of diabetes mellitus is refuted. The history of using longterm drugs (steroids) was denied, no history of drinking alcohol.

On the examination of vital signs, awareness of compos mentis was obtained, blood pressure $120 / 70 \mathrm{mmHg}$, frequency 92 times per minute, respiratory rate 20 times per minute, temperature $38.5{ }^{\circ} \mathrm{C}$ visual analogue score $2-3$. On physical examination of the eye, pale palpebral conjunctiva was found absent, icteric sclera was absent. In the thorax, both thoraxes are normal, found in the abdomen, soepel, liver palpable $3 \mathrm{~cm}$ below the right rib arch, blunt 
edges, soft consistency, flat surface, found tenderness, normal peristalsis. In both extremitas normal.

The laboratory found hemoglobin $13.3 \mathrm{~g} / \mathrm{dl}$, platelets of $196,000 / \mathrm{mm}^{3}$, leukocytes $5,4,000 / \mathrm{mm} 3$, sedimentation rate $20 \mathrm{~mm} /$ hour, segment neutrophils $76 \%$, direct bilirubin $0.16 \mathrm{mg} / \mathrm{dl}$, indirect $0.27 \mathrm{mg} / \mathrm{dl}$, SGOT $53 \mathrm{U} / \mathrm{I}$, SGPT $79 \mathrm{U} / \mathrm{I}$, urea $21 \mathrm{mg} / \mathrm{dl}$, creatinine $0.4 \mathrm{mg} / \mathrm{dl}$, sodium $142 \mathrm{mmol} / \mathrm{L}$, potassium $3.8 \mathrm{mmol} / \mathrm{L}$, chloride $109 \mathrm{mmol} /$ $\mathrm{L}$, blood sugar as $92 \mathrm{mg} / \mathrm{dl}$. The chest radiograph shows a right pleuropneumonia. On noncontrast and non-contrast abdominal CT scan, there was multiple abscesses in the liver of the right lobe, the largest size being $8.44 \times 6.42 \mathrm{~cm}$.

Patients diagnosed with multiple pyogenic liver abscess were treated at RSUDZA and received bed rest therapy, $1800 \mathrm{kcal} /$ day soft food diet, IVFD $\mathrm{NaCl} 0.9 \% 20$ drops per macro minute, metronidazole drip $500 \mathrm{mg}$ every 8 hours, ciprofloxacin 2x $500 \mathrm{mg}$, intravenously paracetamol 1 gram every 8 hours. The patient was treated for 17 days, on the 10th day of hospitalization the patient was performed a laparotomy and multiple incisions of the liver abscess, purulent abscess fluid mixed with blood. Abscess fluid culture was performedthe result is Entamoeba Colli. During the patient's care, the patient experienced clinical improvement, the patient was opened surgical sutures on the 10th and 15 th day after the lapratomy, the surgical wound improved, the patient experienced clinical improvement, currently the patient is still in control at the Internal Medicine and Surgery Department at Cut Nyak Dhien Meulaboh Hospital.

\section{Discussion}

Symptoms of amoebic liver abscess are right upper abdominal pain felt in $75-90 \%$ of patients, many patients seen with acute clinical presentation and pain more severe than pyogenic especially in the right upper quadrant. Sometimes pain is accompanied by nausea, vomiting, anorexia, weight loss, fatigue and painful enlargement of the liver. Spontaneous right upper abdominal pain accompanied by clinical features is found. Episgastric pain is usually seen in amoebic liver abscess in the left lobe. The fever is usually between 38-40 oC, the pattern is intermittent. Malaise, myalgia and atralgia are common. $20 \%$ of patients with suspected amoebic liver abscess have a history of diarrhea or dysentery. Pulmonary symptoms and signs may occur, coughing without phlegm and pleuritic pain can be seen in amoebic liver abscess. But pericardial rub and peritonitis are rare.Left lobe abscess may present with toxemia, severe jaundice and encephalopathy, there is usually a multiple percentage of cases in $5 \%$ of cases. Jaundice is rare and if present indicates a poor prognosis. Ascites indicates inferior vena cava obstruction, and coughing with excessive sputum indicates a ruptured abscess in the right lower lobe of the bronchus. The most common cause is Entamoeba hystolitica (Sharma, M.P. 2003; Nusi, I.A. 2014; Wuerz, T. 2012; Lübbert, C. 2014).

Pyogenic liver abscess is a suppurative process that occurs in liver tissue caused by bacterial invasion through the bloodstream, biliary system or direct penetration. Early symptoms at the onset of pyogenic liver abscess are nonspecific and include malaise, nausea, anorexia and weight loss, headache, myalgia, and arthralgia. In most cases, these prodromal symptoms may be present for several weeks prior. Specific symptoms, such as fever, chills and abdominal pain, although the pain is not always localized to the right upper quadrant. Abscesses adjacent to the diaphragm can cause pleuritic-type pain, cough and dyspnea, and septic shock may occur in some patients, especially in biliary tract obstruction. The clinical presentation of multiple abscesses is usually acute and usually the primary cause is known. Physical examination revealed an enlarged liver accompanied by right upper quadrant pain, 
jaundice was found when the disease was advanced. The presence of a right lung abnormality in the form of pleural effusion is usually a pyogenic liver abscess in the superior segment of the right lobe.

Risk factors for liver abscess are alcoholism, malignancy, HIV infection, malnutrition, use of corticosteroids, immune system disorders, homosexuality, traveling or living in tropical areas. On physical examination, he found an increase in temperature, enlarged liver and tenderness. Aspirate the amoebic abscess with a slightly smelly reddish-brown paste. Tropozoites are only found in $20 \%$ of aspirates. Abnormal chest X-ray results obtained in 50 $80 \%$ of patients with a picture of right lower lobe lung atelectasis, right pleural effusion and increased right hemidiaphragm (Dutta, A. 2012).

Diagnosis of amoebic and pyogenic liver abscess in the laboratory shows leukocytosis, anemia, neutrophilia and an increased sedimentation rate. There may also be an increase in liver function and alkaline phosphatase. The most common cause of amoebic liver abscess is E. Hystolitica. The most common cause of pyogenic liver ambses is E. colliand Kleibsella was the most widely isolated bacteria in the gram-negative aerobic bacteria group. The organism that causes pyogenic liver abscess in western countries is most often E. Colli while in Asia the most common organism is Kleibsella (Dutta, A. 2012; Dhanapal, P.V. 2017; Reyna-fabián, M.E. 2016).

Imaging examination is an important modality for diagnosing liver abscess. Abdominal ultrasound is the main choice for initial diagnosis because it is non-invasive and has a high sensitivity (80-90\%) for obtaining hypoechoic lesions with intermal echoes. Contrast CT scan is used primarily to diagnose smaller abscesses, which can view the entire peritoneal cavity which may provide information about the primary lesion. MRI does not have a higher sensitivity than CT scan. Computed tomography (CT) scan is $95 \%$ sensitive and more accurate than ultrasound in differentiating a liver abscess from other liver lesions. The liver abscess image on CT scan shows a low density image, the use of contrast indicates peripheral enhancement.Liver imaging cannot differentiate between amoebic and pyogenic liver abscess. Percutaneous aspiration of aspiration is required to differentiate between amoebic and pyogenic liver abscess (James S. Dooley, 2011).

Serological tests that can be used include ELISA, indirect hemaglutination, cellulose acetate precipitin, counterimmunoelectrophoresis, immunofluoroscent antibody and rapid agglutination tests. The results of serorological tests should be interpreted clinically by the patient because serum antibody levels remain high for several years after improvement or recovery. The test sensitivity is $95 \%$ and the specificity is more than $95 \%$ (Nusi, I.A. 2014).

There are 4 effective management modalities, namely: 1. Antibiotic therapy, 2. Ultrasound guided aspiration and antibiotics, 3. Percutaneous catheter drainage and antibiotics, 4. Laparatomy, drainage and antibiotics. Liver abscess aspiration is not indicated for diagnostic or therapeutic purposes. Indications for aspiration of liver abscess are: no clinical improvement within 48-72 hours of antibiotic therapy, left lobe liver abscess associated with high mortality and frequency of leakage into the peritoneum or pericardium, large abscesses with a cavity size of more than $5 \mathrm{~cm}$ are at risk of rupture or show signs. compression, surrounding liver tissue, thin-walled abscess $(<10 \mathrm{~mm})$, seronegative abscess, failed non-invasive (medical) treatment, after 4-5 days. Percutaneous drainage is performed on abscesses that are thick-walled and cannot be aspirated or ultrasound guided aspiration fails. Surgical intervention is performed with open surgical drainage, the indication is rare, and is performed in the following conditions: large abscess with failed aspiration or percutaneous 
drainage, clinical worsening after aspiration, amoebic liver abscess with complications such as abscess rupture in the peritoneal cavity with clinical symptoms of peritonitis, rupture abscess in the pleural cavity or pericardial cavity adjacent to the viscera (Zhou, Y. 2010).

Medical therapy for amoebic liver abscess starting with metronidazole $3 \times 750 \mathrm{mg}$ orally for 7-10 days or long-acting nitoimidazole ( 2 gm tinidazole orally and 2 gm ornidazole oral) has been reported to be effective as a single dose therapy. Therapy is then continued with lumenalamubicide preparations to eradicate cysts and prevent further transmission, namely iodoquinol 3x65m mg for 20 days, diloxanide furuote 3 x $500 \mathrm{mg}$ for 10 days, aminosidine (Paramomicin 25-35 mg / kgbb per day, TID for \& -10 days. More than $90 \%$ of patients had a good response to metronidazole therapy by assessing the reduction in pain and fever within 72 hours.

The principles of management of pyogenic liver abscess are administration of antibiotics, percutaneous drainage, drainage by laparotomy. Before there were culture results, broad-spectrum antibiotics were given. Aminoglycosides and ampicillin are given when the source of infection is bile. Third generation cephalosporins are an option if the source of infection comes from the intestine. Metronidazole is given to all pyogenic liver abscesses from various sources of infection to treat anaerobic infections. Percutaneous drainage was performed under ultrasound guidance on abscesses $>5 \mathrm{~cm}$ in size, using indwelling drainage catheters. In multiple abscesses, only the large abscess needs to be aspirated. Abscesses are enough with antibiotics. Surgical drainage is performed on a pyogenic liver abscess that has failed after percutaneous drainage, icteric that does not heal (Dutta, A. 2012; Singh, S. 2013; Waleleng, B. 2014).

Without therapy the abscess will expand into the diaphragm or rupture into the peritoneal cavity. Rupture of the abscess into the thoracic region will cause: hepatobronchial fistula, lung abscess, empyema, sepsis and thrombosis. Thrombosis can occur in the portal vein or hepatic vein due to anaerobic infection. In the abscess rupture into the pericardium causes heart failure, pericarditis, cardiac tamponade, the peritoneum causes: peritonitis, ascites. Other complications are fulminant liver failure, hemobilia, inferior vena cava obstruction, BuddChiari syndrome, cerebral abscess (hematogenous spread): 0.1\% (Waleleng, B. 2014; Nusi, I.A. 2014).

In case I, a 52-year-old male patient presented with complaints of upper right abdominal pain since 1 month ago, worsening in the last 1 day. Fever has been complained of since 1 month ago, fever fluctuates indefinitely, comes down with fever-reducing drugs, complained of weakness. There is nausea and vomiting, vomiting 1-2 times per day, black vomit like coffee is not there. Complained about weight loss. BAK is like strong tea. Liquid defecation has been complained of since 5 weeks ago, initially liquid defecation was accompanied by mucus and blood with a frequency of 3-4 times per day for 3 days, at that time the patient went to the health center and complaints were reduced and now liquid CHAPTER 1-2 times a day is yellow, liquid, no mucus, no blood for the past 1 month. The patient is an agricultural instructor with his daily activities going down to the farm. History.

On vital examination, there was awareness of compos mentis, blood pressure 110/70 $\mathrm{mmHg}$, pulse rate 100 times per minute, breath frequency 24 times per minute, temperature $38.9^{\circ} \mathrm{C}$ visual analogue score $3-4$, underweight body mass index. On physical examination, the eyes of the conjunctiva of the inferior palpebra were pale and absent, the icteric sclera was absent. Both lungs were found to be normal. In the abdomen there is a soepol, the liver is palpable $5 \mathrm{~cm}$ below the right rib arch, $6 \mathrm{~cm}$ below the xiphoid process, there is pressure, flat 
surface, supple consistency, blunt edges, spleen and renal are not palpable, tympanic and peristalsis are present.

On laboratory examination, there was mild anemia, leukocytosis, increased segment neutrophils, kidney function, liver function, albumin, electrolytes and normal blood sugar levels. On ultrasound examination of the liver, multiple mixed echoic lesions were seen in the right and left lobe of the liver with the impression of a liver abscess. On the abdominal CT scan, the impression of a liver abscess was $16.5 \mathrm{~cm}$ in size.

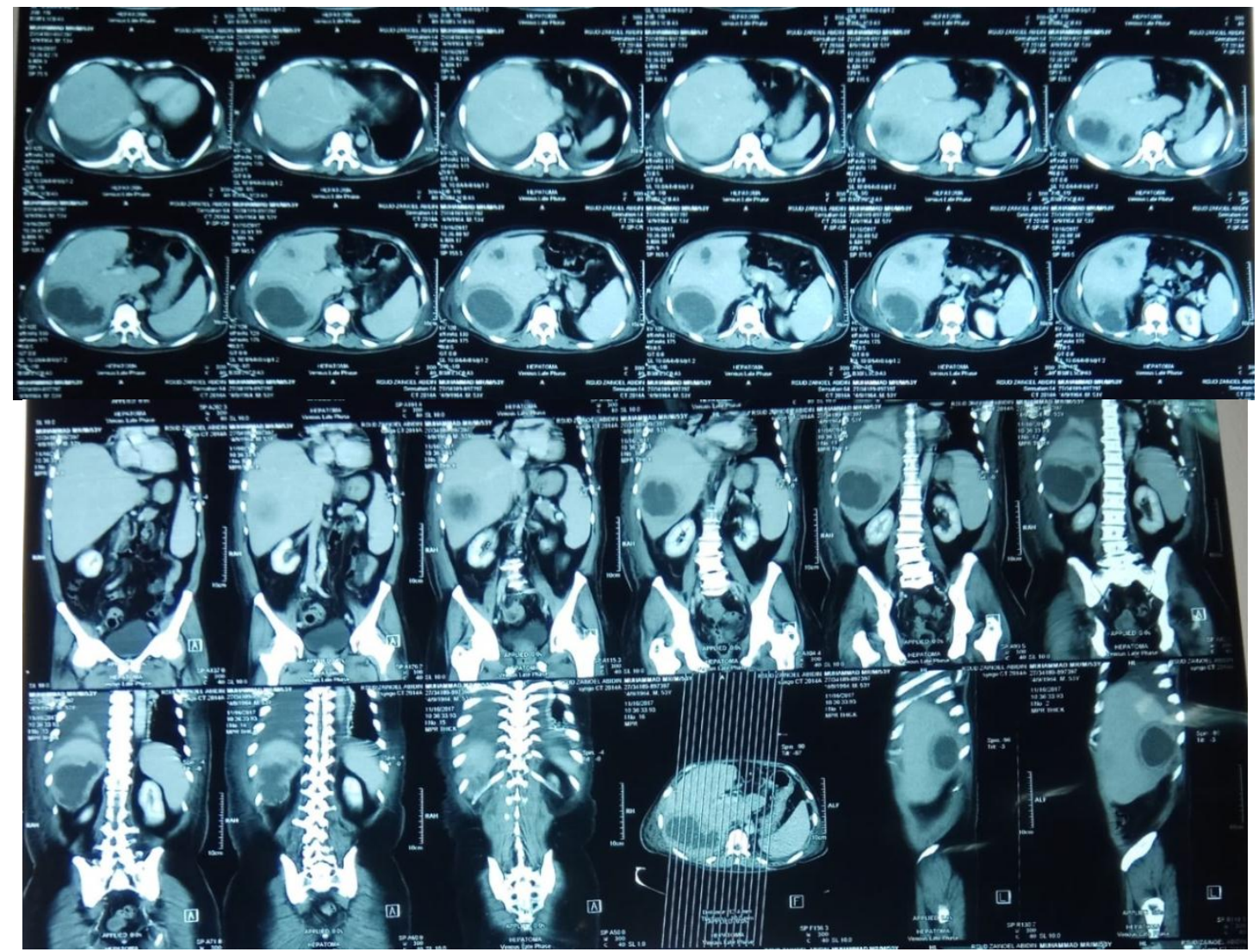

Figure 1.CT Scan Abdomen Amoebic Liver Abscess Size $16.5 \mathrm{~cm}$

The patient was diagnosed with amoebic liver abscess, treated for 20 days, with a diagnosis of amoebic liver abscess, the patient received bed rest therapy, liver diet I $1700 \mathrm{kcal}$ / day, IVFD $\mathrm{NaCl} 0.9 \% 20$ drops per minute, intravenous ceftriaxone $2 \mathrm{~g} / 24$ hours, metronidazole drip $500 \mathrm{mg} / 8$ hours, intravenous ondancetron $4 \mathrm{mg} / 8$ hours, coditam 3x10 $\mathrm{mg}$. The patient was drained of the liver abscess by digestive surgery on the 7 th day of admission, $200 \mathrm{cc}$ of copper red discharge came out of the drainage on the first day, $50 \mathrm{cc}$ on the second day. The results of liver abscess drainage fluid culture showed Entamoeba Hystolitica, metronidazole antibiotic therapy was continued until day 20, the patient during treatment experienced clinical improvement and no complications were found. The patient then went for outpatient treatment, control to the Internal Medicine Department, gastroenterohepatology and Digestive surgery division. 


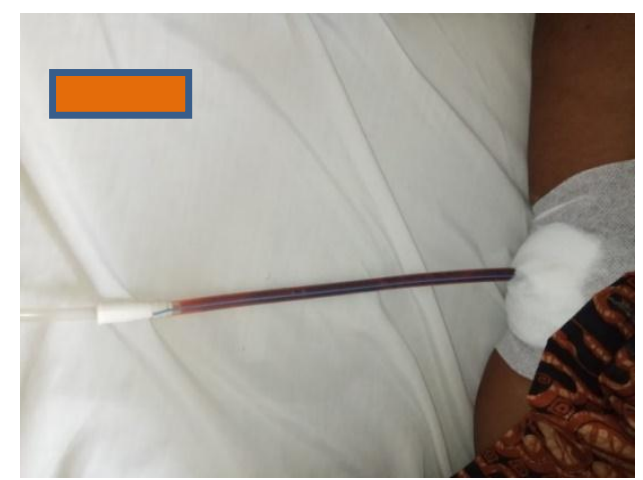

Figure 2. A Dark Red Liver Abscess Drainage

In case II, pA 41-year-old male patient presented with complaints of upper right abdominal pain since 3 months worsening 1 week before admission to hospital. Complained of fever for 3 months is intermittent. Nausea was found, vomiting was found 1-2 times per day. Decreased appetite, weight loss, $4 \mathrm{~kg}$ in the last 3 months. The patient is a trader who is also a farmer. The patient 2 months ago was treated with the same complaint and was diagnosed with a liver abscess. Patients with small liver abscesses measuring $4 \mathrm{~cm}$, the patient received antibiotic therapy during treatment. The patient experienced improvement so that he was discharged with outpatient control at the Internal Medicine Department of RSUDZA. After 1.5 months of the outpatient control patient, the fever returned and the upper right abdominal pain was getting worse so that the patient was hospitalized again.

On vital sign examination, compos mentis awareness was obtained, blood pressure $120 / 70 \mathrm{mmHg}$, frequency 92 times per minute, breath frequency 20 times per minute, temperature $38.5 \mathrm{oC}$, visual analogue score 2-3. On physical examination of the thorax, both thoraxes were normal, found in the abdomen, the liver was palpable $3 \mathrm{~cm}$ below the right arch of the ribs, blunt edges, soft consistency, flat surface, tenderness was found, peristalsis was normal. In the laboratory, there is mild anemia, normal leukocytes, an increase in the sedimentation rate of $20 \mathrm{~mm}$ / hour, liver function, kidney, electrolytes, normal. On chest Xray, he found right pleuropneumonia. On non-contrast and non-contrast abdominal CT scan, there was multiple abscesses in the liver of the right lobe with the largest size being $8.44 \times 6.42$ $\mathrm{cm}$.

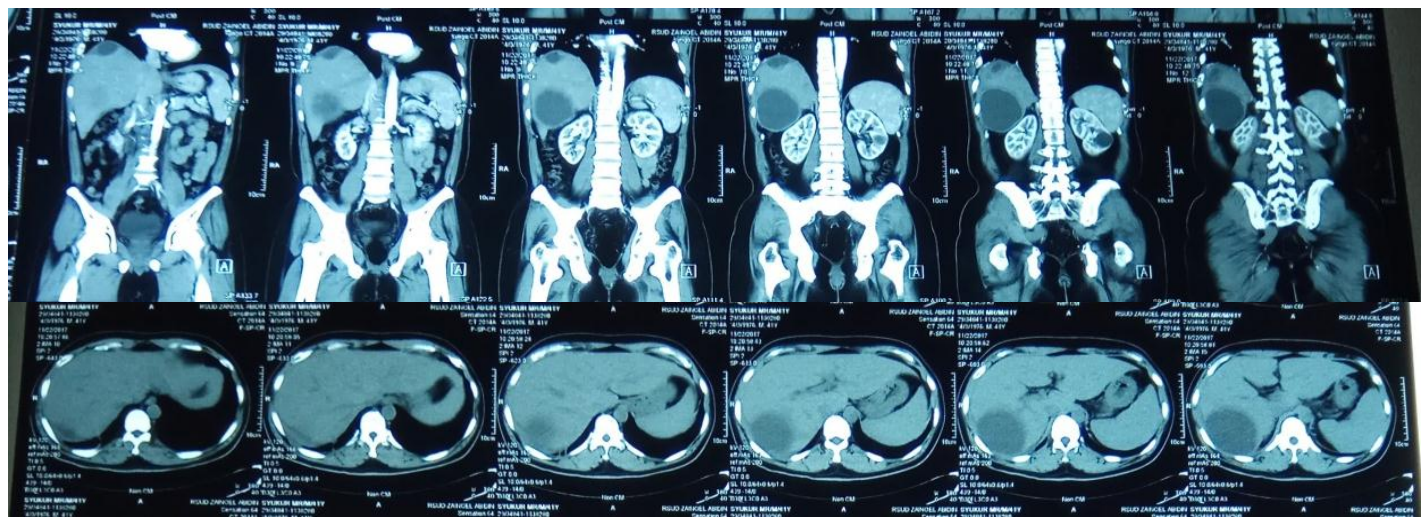

Figure 3. Contrast CT Scan of Multiple Abscesses in the Liver of the Right Lobe with The Largest Size being $8.44 \times 6.42 \mathrm{~cm}$

Patients diagnosed with multiple pyogenic liver abscess were treated at RSUDZA and received bed rest therapy, $1800 \mathrm{kcal} /$ day soft food diet, IVFD $\mathrm{NaCl} 0.9 \% 20$ drops per macro minute, metronidazole drip $500 \mathrm{mg}$ every 8 hours, ciprofloxacin 2x $500 \mathrm{mg}$, intravenously paracetamol 1 gram every 8 hours. The patient was treated for 17 days, on the 
10th day of hospitalization the patient was performed a laparotomy and multiple incisions of the liver abscess, purulent abscess fluid mixed with blood. The abscess fluid was cultured as a result of Entamoeba Colli, and metronidazole antibiotic therapy was continued. During treatment, the patient experienced clinical improvement, the patient was opened surgical sutures on the 10th and 15th day after laparotomy, the surgical wound improved, the patient experienced clinical improvement, currently the patient is still a control at the Internal Medicine and Surgery Department at Cut Nyak Dhien Meulaboh Hospital.

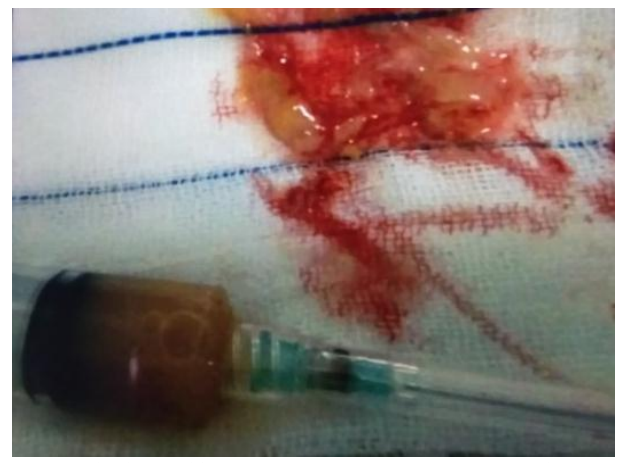

Figure 4. Solid Yellow Pyogenic Liver Abscess Fluid

\section{Conclusion}

1. The principles of management of pyogenic liver abscess are administration of antibiotics, percutaneous drainage, drainage by laparotomy.

2. Without therapy the abscess will expand into the diaphragm or rupture into the peritoneal cavity. Rupture of the abscess into the thoracic region will cause: hepatobronchial fistula, lung abscess, empyema, sepsis and thrombosis. Thrombosis can occur in the portal vein or hepatic vein due to anaerobic infection.

3. Medical therapy alone is effective in most cases of amoebic liver abscess, while some forms of intervention usually require pyogenic liver abscess. The mortality rate is lower in amoebic liver abscess than in pyogenic liver abscess. A multidisciplinary approach, including gastroenterologists, radiologists and surgeons, as well as laboratories, is essential for the successful treatment of a curable liver abscess.

\section{References}

Dutta, A. \& Bandyopadhyay, S. Management of Liver Abscess. 22, 469-475 (2012). Nattakom, S. et al. Amebic Liver Abscesses Masquerading as Pyemic Abscesses. 33, 145-147 (2017).

Singh, S. et al. Treatment of liver abscess : prospective randomized comparison of catheter drainage and needle aspiration. 1-8 (2013).

Mortele, K. J., Segatto, E. \& Ros, P. R. The Infected Liver: OBJECTIVES. 937-955 (2004).

Waleleng, B. . Buku Ajar Ilmu Penyakit Dalam. in (ed. Setiati, S.) 1996-1999 (Interna Publishing, 2014).

Sharma, M. P. \& Ahuja, V. Amoebic Liver Abscess. 4, 107-111 (2003).

Chiari, B., Chiari, B., Jenks, P.- \& Syndrome, B. C. Amoebic liver abscess The mechanisms underlying hepatitis $C$ virus genotype 3-mediated liver damage. 39, 291-292 (2003).

Nusi, I. A. Buku Ajar Ilmu Penyakit Dalam. in (ed. Setiati, S.) 1991-1995 (2014).

Wuerz, T. et al. A review of amoebic liver abscess for clinicians in a nonendemic setting. 26, 729-733 (2012).

Lübbert, C. \& Karlas, T. Therapy of Liver Abscesses. 334-341 (2014). doi:10.1159/000366579

Dhanapal, P. V \& Banurekha, R. Clinical profile of liver abscess in a tertiary referral hospital. 
4, 2025-2029 (2017).

Reyna-fabián, M. E. et al. Analysis of the Bacterial Diversity in Liver Abscess : Differences between Pyogenic and Amebic Abscesses. 94, 147-155 (2016).

James S. Dooley, Anna S. F. Lok, Andrew K. Burroughs, 2011. Sherlock's Diseases of the Liver and Biliary System. (2011). doi:10.1017/CBO9781107415324.004

Zhou, Y. \& Li, G. Diagnosis and management of complicated intra-abdominal infection in adults and children: Guidelines by the Surgical Infection Society and the Infectious Diseases Society of America. Chinese J. Infect. Chemother. 10, 241-247 (2010).

Christian, A, M. et al. (2020). Epidemiological profile of Ascariasis in Provincial General Reference Hospital of Kinshasa, Democratic Republic of the Congo. Britain International of Exact Sciences (BIoEx) Journal Vol. 2 (2): 541-546. 